\title{
Silicone Oil Tamponade Hydrostatics and Technology for Additional Mechanical Support of Retina
}

\author{
DOI: $10.17691 / \mathrm{stm} 2018.10 .4 .02$ \\ Received March 24, 2018
}

E.G. Kazimirova, PhD Student ${ }^{1}$; Physicist, Department of Physics of Polymers and Crystals, Faculty of Physics ${ }^{2}$;

V.V. Shiryaev, PhD, Lecturer, Department of General Physics;

P.V. Lyskin, MD, PhD, Ophthalmic Surgeon, Department of Vitreoretinal Pathology and Eye Diabetes ;

G.V. Stepanov, Head of the Laboratory of Magnetic Pigments and Composites"; Junior Researcher,

Department of Physics of Polymers and Crystals, Faculty of Physics;

E.Yu. Kramarenko, DSc, Professor, Department of Physics of Polymers and Crystals, Faculty of Physics ${ }^{2}$

${ }^{1}$ S. Fyodorov Eye Microsurgery Federal State Institution, 59a Beskudnikovsky Blvd., Moscow, 127486, Russia;

2Lomonosov Moscow State University, 1 Leninskiye Gory, Moscow, 119991, Russia;

${ }^{3}$ Moscow Institute of Physics and Technology (State University), 9 Institutskiy per., Dolgoprudny,

Moscow Region, 141701, Russia;

${ }^{4}$ State Scientific Research Institute of Chemistry and Technology of Organoelement Compounds,

38 Entuziastov Shosse, Moscow, 111123, Russia

Silicone oil tamponade is widely used in the surgical treatment of retinal detachments. The article provides analysis of mechanical forces exerted to the retina in various parts of the vitreous cavity. There are areas of tamponade effect insufficiency, in which it may be beneficial to create additional means which would support the retina keeping it attached to the pigment epithelium.

The aim of the study was to develop a new technique desired to keep the retina attached in the areas where retina lacks effective tamponade effect of silicone oil. The technology is desired to be implemented in challenging cases of retinal detachments caused by giant retinal breaks, retinodialysis, or in patients with hard-to-remove epiretinal membranes in proliferative vitreoretinopathy.

Materials and Methods. The proposed technique - magnetic scleral buckling - is able to exert pressure to the retina by the means of mutual magnetic attraction of the magnetic scleral buckle (outer buckle) sutured to the retina and endovitreal magnetic buckles (inner buckles) made of magnetoactive elastomer and placed onto the retina in the projection of the outer magnetic buckle. A series of ex vivo experiments was performed to elaborate the technique using isolated cadaver donor eyes: we performed magnetic scleral buckling and then subtotal vitrectomy with iatrogenic detaching of retina followed by implantation of endovitreal magnetic buckles (inner buckles) made of magnetoactive elastomers to provide additional mechanical support for the retina.

Results. Elastic properties of the magnetic scleral buckles didn't differ from those of common scleral buckles. Endovitreal magnetic buckles fitted well onto the retina, smoothly, tightly and effectively held it pressing against the pigment epithelium. We tried buckles of various sizes and shapes and chose the best fitted for endovitreal manipulations. Endovitreal buckles securely supported the retina and could be easily removed with $25 \mathrm{G}$ vitreotome.

Conclusion. The developed technique of magnetic scleral buckling allowed to keep retina securely reattached with magnetic forces as was shown during ex vivo experiments. Further in vivo studies of the technique are required.

Key words: silicone oil; vitreous tamponade; complicated retinal detachment; retinodialysis; giant retinal breaks; mechanical support of retina; magnetic buckles; magnetic scleral buckling; magnetoactive elastomers.

\section{Introduction}

Physical basis of silicone oil tamponade. Silicone oil tamponade of the vitreous cavity is widely used in surgical treatment of retinal detachments. One of the characteristic features of silicone tamponade is the impossibility to completely fill the vitreous cavity with silicone. Once tamponade is performed there are still some areas of retina lacking tamponade effect: light silicone floats on top leaving lower retina without tamponade effect; in case of heavy silicone oil is used, upper retina lacks tamponade effect. And the further meniscus (boundary between silicone and aqueous humour) is from the retina, the larger part of retina lacks tamponing effect (Figures 1,2). Leaving some areas of the vitreous without tamponade is less dangerous than excessive tamponade because overfilling of vitreous with silicone leads to uncontrolled raise of intraocular pressure. Tamponade effect can be considered ideal only if $100 \%$ filling of the vitreous with silicone is achieved which is practically impossible to implement. In any case there is still larger or smaller area lacking tamponade effect.

There is a remarkable experiment of Fawcett et al.

Corresponding author: Elena Yu. Kramarenko, e-mail: kram@polly.phys.msu.ru 


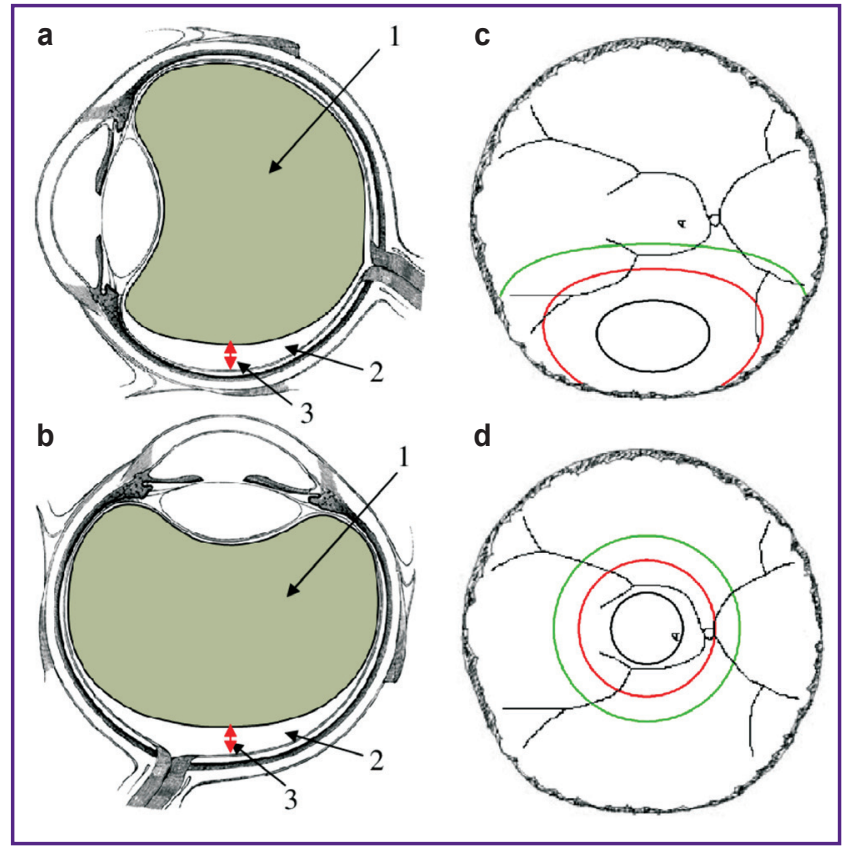

Figure 1. Filling of the vitreous cavity with light silicone oil:

(a) in the standing position; (b) in the supine position; 1 silicone oil; 2 - residual non-tamponed area of the vitreous cavity; 3 - height of the meniscus level; (c), (d) area of the retina, devoid of the tamponade effect: in black - the silicone meniscus is at a distance of $1 \mathrm{~mm}$, in red - of $2 \mathrm{~mm}$, and in green - of $3 \mathrm{~mm}$

on filling model vitreous cavity with silicone which proved that $100 \%$ filling is not possible due to physical properties of silicone oil [1].

It was found [2] that even when vitreous cavity is slightly underfilled with silicone, significant area of the retina lacks tamponade effect (see Figures 1, 2). Thus if meniscus is $2 \mathrm{~mm}$ away from the retina, the surface of the retina lacking tamponade effect is $3.8 \mathrm{~cm}^{2}$, while if meniscus is $3 \mathrm{~mm}$ away from the retina - it grows to $6.5 \mathrm{~cm}^{2}$. Therefore even when vitreous chamber appears to be filled with silicone almost completely, spherical shape of the eye ball results in the fact that even slight underfill of the vitreous leads to significant part of the retina lacking tamponade effect.

Silicone tamponade is a kind of trade-off with respect to achievement of necessary tamponade effect in all areas of the vitreous cavity and yields good results in cases of retinal breaks are located only in one retinal hemisphere (upper retina or lower retina). Retinal breaks locating in both upper and lower retina rule out secure tamponade.

To assess possibility of achieving secure tamponade effect in all areas of the vitreous, it's worth considering physical forces acting on the retina during silicone oil tamponade. Literature search gained no description of silicone oil tamponade devoted to understanding of the

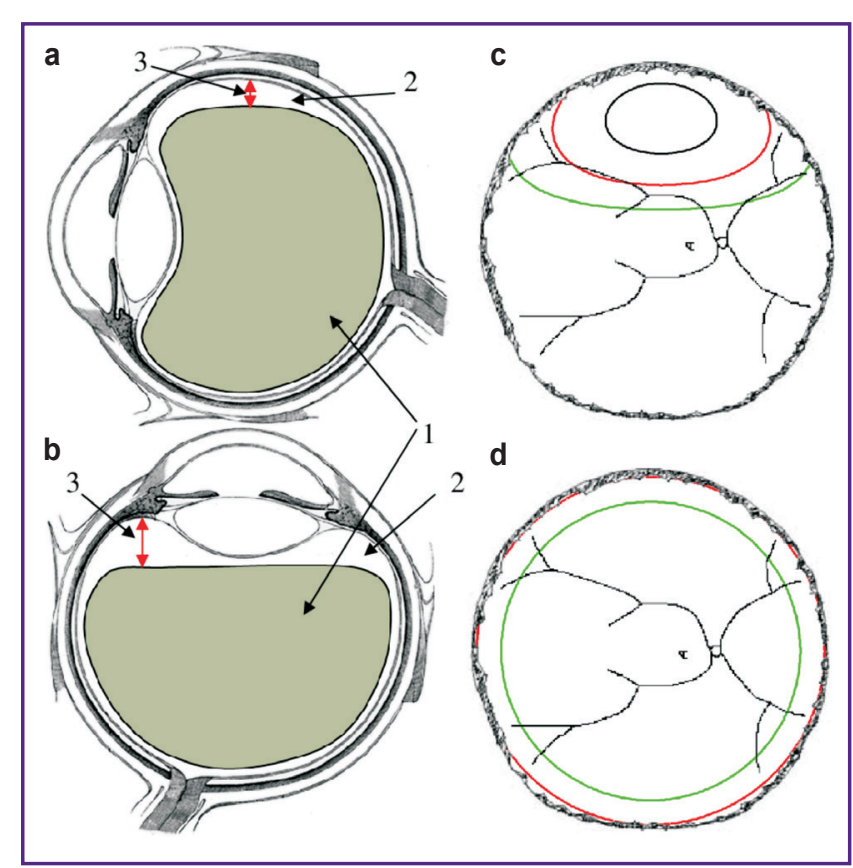

Figure 2. Filling of the vitreous cavity with heavy silicone oil:

(a) in the standing position; (b) in the supine position; 1 silicone oil; 2 - residual non-tamponed area of the vitreous cavity; 3 - height of the meniscus level; (c), (d) area of the retina, devoid of the tamponade effect: in black - the silicone meniscus is at a distance of $1 \mathrm{~mm}$, in red - of $2 \mathrm{~mm}$, and in green - of $3 \mathrm{~mm}$

physical processes underlying the tamponade. However this problem deserves more thorough consideration because silicone oil tamponade remains a widely used technique and insufficient understanding of its physical laws hinders its maximally efficient use in clinical practice.

Let us consider tamponed vitreous chamber and calculate pressure forces pushing the retina towards pigment epithelium and holding it in normal anatomical position (Figure 3, z-axis is directed upwards with fundus being at zero).

In case of light silicone, pressure acting on the retina at height equals (considering pressure at the top of the eye as zero):

$$
P=\rho_{\text {So }} g(D-z),
$$

where $\rho_{s o}$ is silicone specific density, $g$ is gravitational acceleration, and $D$ is the diameter of eye.

In case of heavy silicone things are a bit more complicated. Pressure in aqueous humour above meniscus is

$$
P=\rho_{A H} g(D-z),
$$

where $\rho_{A H}$ is specific density of the aqueous humour. While crossing from the aqueous humour to the tamponing agent pressure experiences a step-change due to the surface tension: 


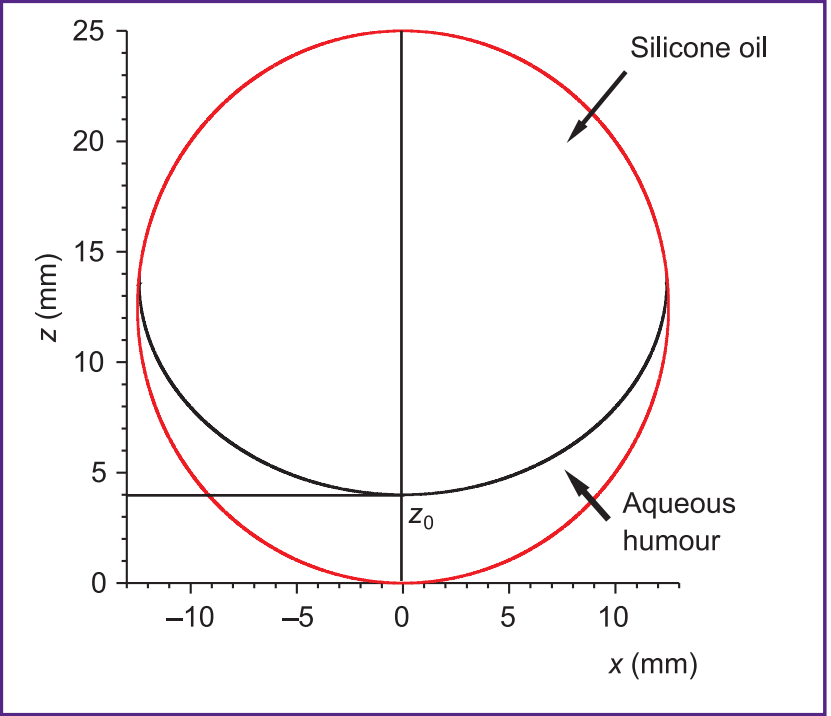

Figure 3. Schematic representation of the vitreous cavity, filled with light silicone oil, and the coordinate system imposed on it; the bottom of the eye is taken zero; $z_{0}-$ coordinate of the meniscus

$$
\Delta P=2 \frac{\sigma_{S O-A H}}{R_{0}},
$$

where $\sigma_{S O}-A H$ is the surface tension at the boundary between silicone oil and aqueous humour, and $R_{0}$ is an average radius of curvature of meniscus at its top $(x=0)$.

Then pressure grows again linearly proportionally to the silicone oil density:

$$
P=\left\{\begin{array}{l}
\rho_{A H} g(D-z), \text { where } z>z_{0} ; \\
\rho_{A H} g\left(D-z_{0}\right)+2 \frac{\sigma_{S O-A H}}{R_{0}}-\rho_{S O} g\left(z-z_{0}\right), \text { where } z<z_{0},
\end{array}\right.
$$

where $z_{0}$ is coordinate of the meniscus.

Pressure dependence on height for both cases above is plotted on Figure 4. The value of $R_{0}$ was calculated with hydrostatic model similar to that in [3].

Figures 5,6 present the distribution of the pressure pushing the retina down in both cases of light and heavy silicone oil in more intuitive form.

As pressure distribution diagrams show, pressing effect on the retina from the silicone oil exists in lower vitreous only (in a standing position) while it vanishes to zero when approaching the upper areas; and this holds for light as well as heavy silicone oil. This can be explained by the fact that specific gravity of the silicone oil is virtually the same as that of the water $(0.95-$ $0.98 \mathrm{~g} / \mathrm{cm}^{3}$ for light silicone and $1.02-1.06 \mathrm{~g} / \mathrm{cm}^{3}$ for heavy silicone). Holding of the retina in normal anatomic position during silicone tamponade is provided exclusively by the fact that silicone oil that fills the vitreous, being hydrophobic, displaces water medium away from the retinal breaks and thus prevents liquid from entering underneath the retina through the breaks. Meanwhile light silicone oil does this in upper retina while

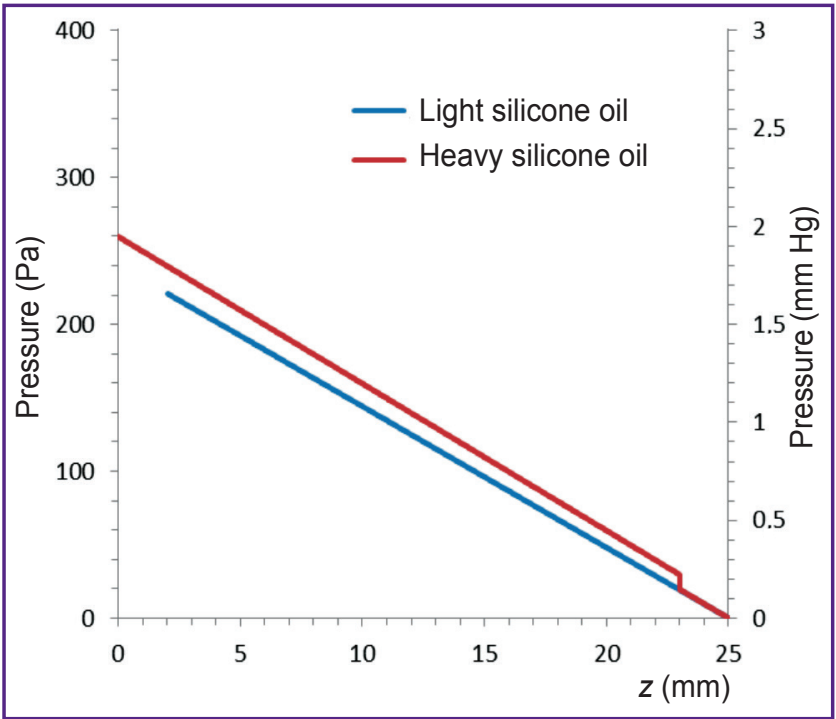

Figure 4. Pressure exerted on the retina at different values of height $z$ in the cases of light and heavy silicone oil in the supine patient position

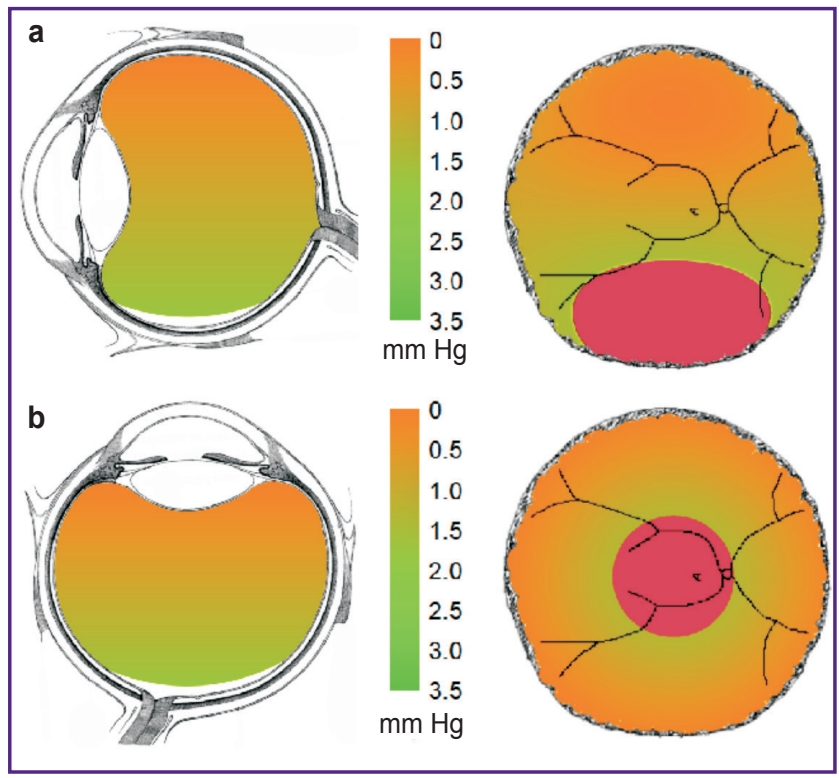

Figure 5. Pressure distribution in the retina under light silicone oil tamponade of the vitreous cavity in the case of circular retinotomy:

(a) in the standing position; (b) in the supine position; left general view of a tamponed vitreous cavity; right - schematic depiction of the fundus; in red - retina in the zone of nontamponed vitreous cavity

heavy silicone oil - in lower retina. But silicone oil does almost not press the retina against pigment epithelium, thus silicone oil tamponade can be effective exclusively when all the tractions are completely resolved and all the breaks are of small or intermediate size. In case of any traction effect on the retina close to the break, just the 


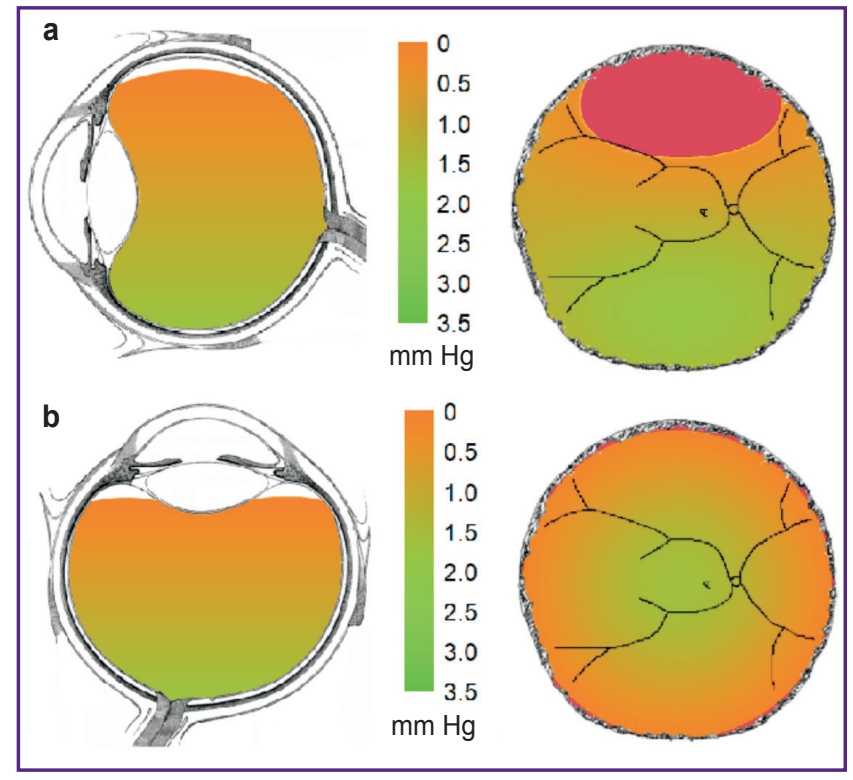

Figure 6. Pressure distribution in the retina under heavy silicone oil tamponade of the vitreous cavity in the case of circular retinotomy:

(a) in the standing position; (b) in the supine position; left general view of a tamponed vitreous cavity; right - schematic depiction of the fundus; in red - retina in the zone of nontamponed vitreous cavity

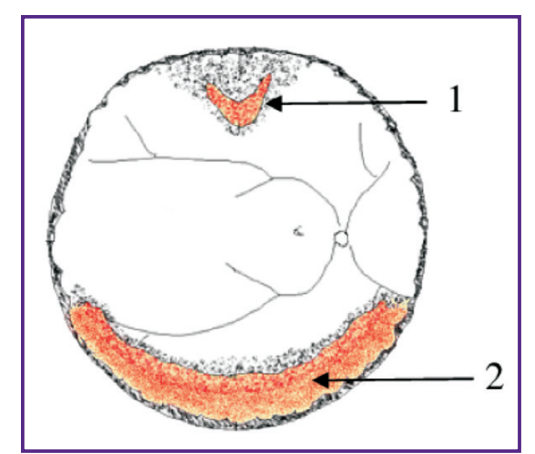

Figure 7. A complicated case of retinal detachment, combining a break in the upper quadrant with the need for lower partial retinotomy:

1 - retinal flap break; 2 - retinotomy zone

displacing of the liquid away from the break would be insufficient. Despite silicone oil presence in the vitreous there will be no tamponade effect because retinal break will be exposed to aqueous humour penetration under the retina; the liquid will still penetrate into subretinal space through the break thus causing retinal detachment reccurence. Therefore when pressing effect on the retina is necessary the silicone tamponade may be insufficient.

Thus in case of retinal breaks and defects localized exclusively in upper or lower retina problem can be solved by light or heavy silicone oil tamponade. Yet there are quite frequent cases requiring secure tamponade effect in all areas of the vitreous: e.g. in cases in which it is not possible to remove epiretinal membranes completely. During such treatment the need of total or subtotal retinotomy arises; and if the clinical case involves breaks in the opposite quadrant of the retina too, there emerges situation hard to be resolved with traditional techniques. Consider one of such complicated cases: e.g. there is a break in upper retina while it is necessary to perform retinotomy in lower quadrants as there is no other opportunity to reattach the retina (Figure 7). Such situations occur sometimes in clinical practice. In such cases surgeon has to decide how to achieve reliable tamponade effect both in upper and lower vitreous simultaneously.

One of the options here is to divide the surgery into two stages: primary surgery to achieve retinal reattachment in just one hemishere of retina (upper or lower retina) and then complete attachment during the secondary surgery [4]. Such two-stage solution would be reasonable once anatomical success was the only purpose of the surgical treatment of the retinal detachment. But once one recalls the functional outcome, the technique above contradicts the fundamental principle: the longer retinal detachment persists, the worse the functional outcome of the treatment will be. In this aspect, this two-stage technique is just a desperate attempt: once it's impossible to achieve normal anatomical position for the entire retina simultaneously and as soon as possible, one has to accept attachment in one hemisphere only. Clearly the most rational approach would be the one allowing reattachment and support of the retina in both upper and lower areas simultaneously. This is possible to achieve in the following way: employ additional opportunities to modify surgical technique, namely, append silicone tamponade with approaches allowing additional support of the retina in the areas with no tamponade effect or in the areas where supporting pressure force exerted to the retina can be insufficient just from the physical point of view. Such mechanical support can be necessary during the surgery not only in case of retinotomy but also in cases in which one cannot completely remove vitreous from the retina in some areas thus exposing them to the risk of future epiretinal fibrosis and possible emergence of the need for future retinotomy.

Proposed technique of mechanical support of the retina. To create additional means of mechanical support of the retina we developed a new technique: magnetic scleral buckling. Magnetic scleral buckling is basically modified technique of scleral buckling widely employed in retinal detachment treatment including its combination with vitreoretinal surgery. It's worth noting that nowadays combined surgeries including endovitreal approach together with scleral buckling and are of growing interest again as there accumulated data showing such combined surgeries increase anatomic success rate in treatment of complicated retinal detachments [5-8].

The technique we propose - magnetic scleral buckling - is able to actively support the retina by means of mutual magnetic attraction of the magnetic 
scleral buckle sutured to the sclera and the endovitreal magnetic buckles (inner buckles) made of magnetoactive elastomer and placed onto the retina in the projection of the scleral magnetic buckle. Scleral magnetic buckle is made of medical silicone and apparently doesn't differ from the common silicone scleral buckle while it contains permanent $\mathrm{NdFeB}$ magnets inside (Figure 8). It may be of various shapes or sizes. After suturing of magnetic scleral buckle and performing vitrectomy we suggest to employ endovitral magnetic buckles (inner buckles) which are essentially thin elastic silicone implants containing ferromagnetic substances. Ferromagnetic substances are surrounded with pure silicone and thus not contacting eye tissues. Mutual attraction of the scleral magnetic buckle and endovitreal magnetic buckles results in effective supporting the retina in normal anatomic position (Figure 9).

Figures 8, 9 demonstrate how to employ the device for circular buckling (cerclage). The circular buckling was chosen as an example. The same way sectoral, bisectoral or radial scleral buckling can be performed as well. Employment of magnetic buckle doesn't disturb principles of scleral buckling technique because it changes neither diameter of the buckle nor its elastic properties: mechanical properties of the magnetic buckle do not differ from those of common scleral buckle.

Need for mechanical support of the retina may arise in cases of retinotomy and presence of residual retinal tractions. Another indication for mechanical support may be cases with incomplete removal of the vitreous from some areas of the retina thus making these parts prone to fibrosis formation and increased risk of future need of additional retinotomy. Surgeons prefer to refrain from unneeded retinotomy avoiding thus additional traumatizing because minimization of traumas is one of the basic surgical axioms. If there is high risk of retinotomy in future, additional mechanical support

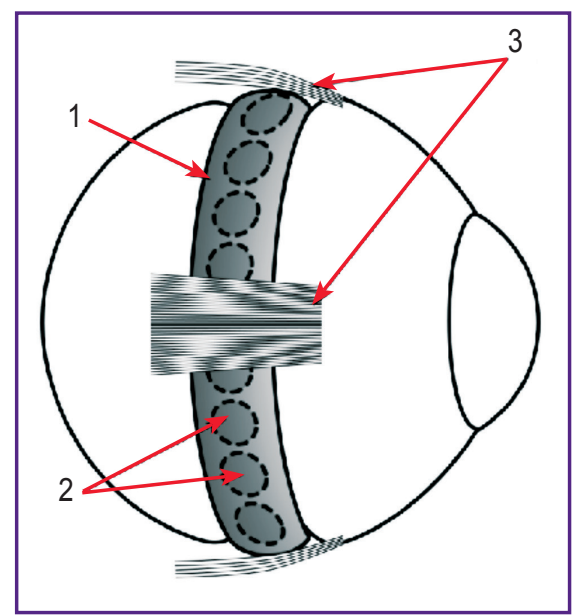

Figure 8. Schematic representation of the eye with a circular scleral indentation caused by a scleral (outer) magnetic buckle:

1 - buckle; 2 - magnets; 3 - eye rectus muscles

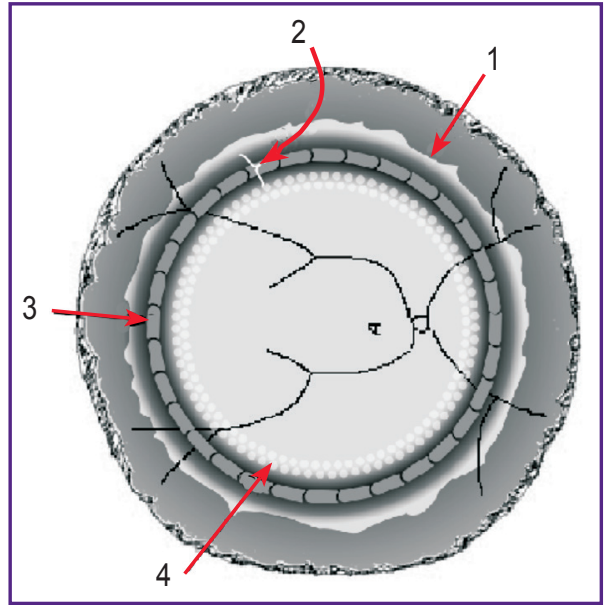

Figure 9. Schematic representation of the eye fundus after circular retinotomy followed by implantation of inner magnetic buckles:

1 - edge of the retina after retinotomy; 2 - indentation formed by outer magnetic outer sutured to the sclera; 3 inner magnetic buckles superimposed on the retina in the projection of the outer magnetic buckle; $4-$ circular laser photocoagulation

may become a decisive factor allowing final avoiding of retinotomy as pressing effect by the endovitral magnetic buckles may withstand traction forces by the newly forming epiretinal membranes. This is not about strong tractions by well-formed epiretinal membranes but in case of just forming epiretinal fibrosis additional mechanical support of the retina may provide some time until reliable chorioretinal attachments form in the area of photocoagulation spots applied during surgery.

The proposed device can exert pressure of up to $3.5 \mathrm{~mm} \mathrm{Hg}$ actively supporting the retina in its normal anatomical position. This pressure can be varied in a wide range by varying content of the endovitreal buckles and size of the magnets in the scleral buckle [9]. Device allows not only effectively press the retina towards the pigment epithelium intrasurgically during the laser coagulation, but also preserving it normal anatomical position in post-op while silicone oil exerts almost no pressing force on the retina (Figures 10, 11).

Need for additional mechanical support may arise not only in cases with persisting tractions caused by proliferative vitreoretinopathy but also in cases of giant retinal break with traction or extensive retinodialysis. Traumatic retinal detachments also pose a challenge for vitreoretinal surgeons. In most cases not complicated by prominent epiretinal fibrosis or presence of gross tractions the problem is likely to be solved by conventional techniques, but there are some situations when it doesn't seem possible to achieve at least partial recovery of vision somehow but with mechanical support of the retina to the underlying tissues. Such cases still occur and sometimes they are impossible to be resolved with conventional techniques. 


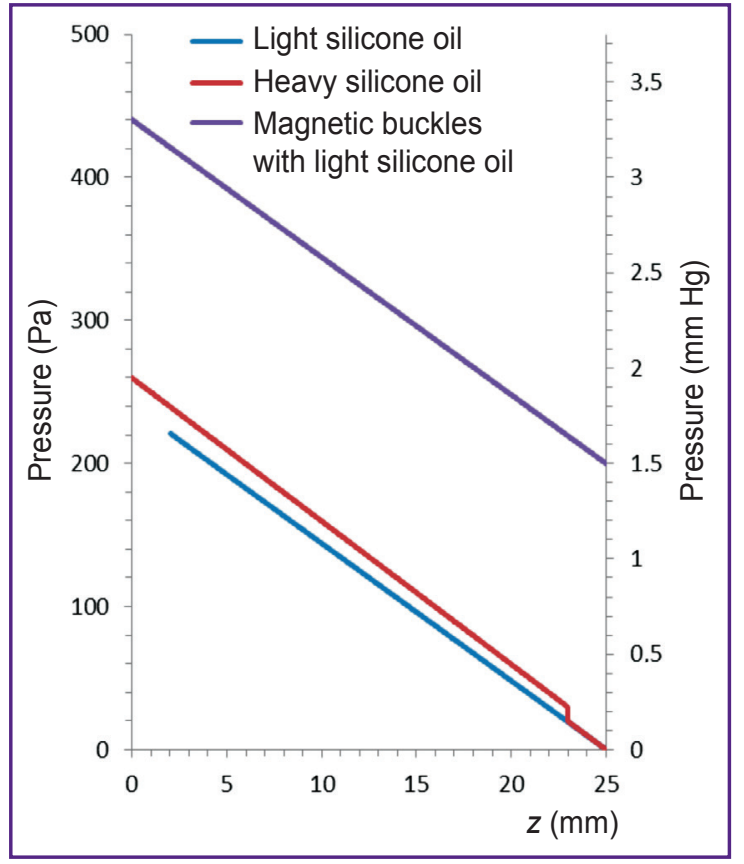

Figure 10. Pressure exerted on the retina at different values of height $z$ in the cases of light and heavy silicone oil in the supine patient position

Also shown is the pressure developing in the presence of a scleral (outer) magnetic buckle with a diameter of $3 \mathrm{~mm}$ and inner buckles with a thickness of $0.2 \mathrm{~mm}$

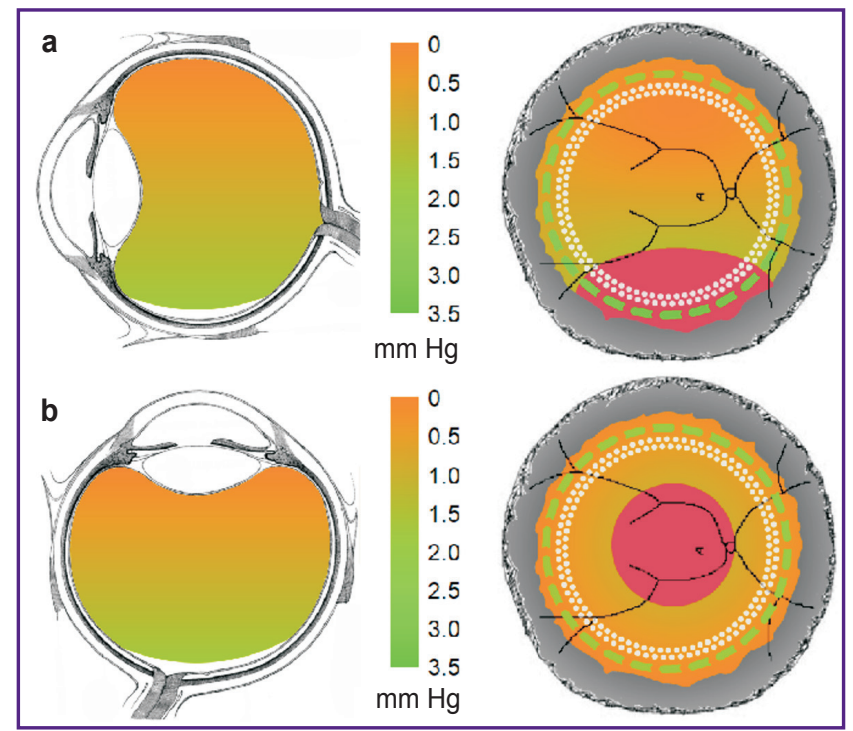

Figure 11. Pressure distribution in the retina in the presence of magnetic buckles combined with light silicone oil tamponade of the vitreous cavity in the case of circular retinotomy

(a) in the standing position; (b) in the supine position

Retinal tacks were suggested previously for mechanical fixation of retina in complicated cases [10]. Retinal tacks helped to hold retina in normal anatomic position thus making photocoagulation possible. In case of extensive break at ora serrata mechanical support helped to attach retina without folds formation [10]. First short-term studies yielded positive outcomes of retinal tacks use [11-14] thus spreading them as a supplementary tool in treatment of complicated retinal detachments. But inefficiency of retinal tacks in retinal fixation in cases of severe proliferatative vitreoretinopathy (PVR) led to rapid abandonment of their use in all cases including less complicated ones [10].

Tendency of retina to curl inside and resist straightening is another technical difficulty sometimes occurring in surgical treatment of giant breaks, traumatic breaks and extensive retinodialysis. Rigid retina may not adhere even when perfluorocarbon liquids (PFCL) are employed. In such patients the only remaining options are retinectomy or mechanical support of retina, probably following preliminary retinectomy. Special attention should be paid to cases requiring circular retinotomy: after its completion the edge of remaining retina often becomes wavy due to residual tractions and retina does not reattach even with PFCL. Such situation renders photocoagulation impossible and thus disallows surgery completion using conventional techniques only.

Suture fixation of retina was suggested in these situations. Takhchidi et al. [15] and Pashtaev [16] performed suture fixation to treat giant breaks and retinodialysis. Edge of retinal defect was fixed as follows: long straight needle was inserted into the vitreous through 23G port with a special coaxial forceps. Then retina was punctured towards and through the sclera at the edge of retinal defect taking the needle to the outer surface of the sclera preliminary cleared of conjunctive. Thread ends were tied with a knot on the outer surface of the sclera [16]. According to authors this technique allowed to repair normal anatomic position of retina in cases of giant breaks and breaks at ora serrata.

However suturing of the retina as well as retinal tacks are not used nowadays. Both techniques had disadvantages, namely low ratio of supported retinal area to the amount of trauma induced (during tack insertion or tissue puncturing) and insufficient secure sealing (insufficient hermeticity). In any case between tacks or sutures there remained a zone of insecure attachment. Another drawback is its traumatizing effect: retinal tacks application poses risk of excessive pressure exerted on the retina with its possible crushing that may not only cause the hemorrhage but also potentially contribute to PVR progression.

In case of the magnetic scleral buckling technique employment the supplementary mechanical retinal support is provided by means of magnetic interactions, so retina is subjected to mild pressing action of the magnetic material of endovitreal buckles. This prevents crushing of the retina during implantation and doesn't require puncturing of the tissues. The technique allows wide-area pushing of the retina and hermetic sealing of retinal breaks. The magnetic scleral buckling 
technique presented allows effective supporting the retina in various challenging situations without additional traumatizing. The magnetic scleral buckling technique may be useful in extremely challenging cases.

Purpose. To develop a new technique allowing supporting the retina in normal anatomic position in the areas where the silicone oil tamponade effect is insufficient. The technique is called magnetic scleral buckling and desired to be used in treatment of complicated retinal detachment with giant breaks, retinodyalisys or hard-to-remove epiretinal membranes in cases of PVR.

\section{Materials and Methods}

We performed a series of experiments on cadaver donor eyes in conditions maximally close to the real life: we employed the same equipment that's routinely used for vitreoretinal surgeries, after suturing scleral magnetic buckles we performed vitrectomy and retinotomy, filled vitreous cavity with PFCL and applied endovitreal magnetic buckles onto the retina. For scleral buckling we employed magnetic buckles $4-5 \mathrm{~mm}$ in diameter containing NdFeB magnetic discs $3 \mathrm{~mm}$ or $4 \mathrm{~mm}$ in diameter and $1 \mathrm{~mm}$ thick placed in a row. Another type of outer magnetic buckles had two rows of $\mathrm{NdFeB}$ magnets $2 \mathrm{~mm}$ in diameter (Figures 12, 13). These buckles allowed local indentation of the sclera (sectorally or radially) or its circular indentation in cases of cerclage similar to that in conventional scleral buckling. We studied not only disc-shaped but also rectangular magnets contained in scleral magnetic buckles as well as scleral magnetic buckles with various magnet polarity orientations.

Additional mechanical support of the retina was provided by implanted endovitreal magnetic buckles experimental samples of magnetoactive elastomer 3-10 mm long, 1-2 mm wide and 0.2-0.4 mm thick. Implants were made of soft elastic silicone elastomer impregnated with ferromagnetic particles less than $20 \mu \mathrm{m}$ in diameter. The particles were completely covered by pure silicone thus preventing ferromagnetic

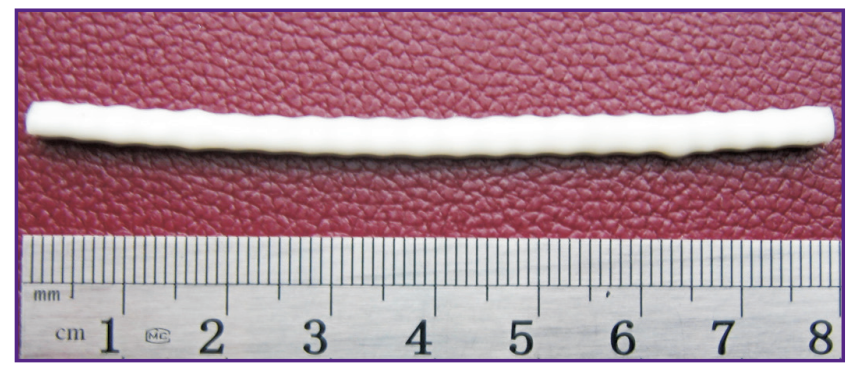

Figure 12. Episcleral magnetic buckle for circular scleral buckling (cerclage)

The buckle is made of medical silicone and contains $3 \mathrm{~mm}$ permanent NdFeB magnets

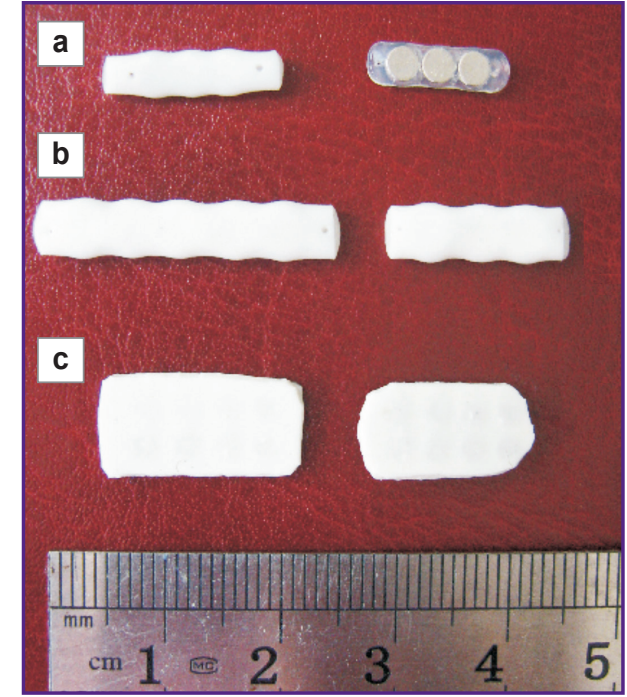

Figure 13. Experimental samples of magnetic scleral buckles for local indentation of the sclera

The buckles contain permanent magnets: (a) with a diameter of $3 \mathrm{~mm}$; (b) with a diameter of $4 \mathrm{~mm}$; (c) two rows of magnets with a diameter of $2 \mathrm{~mm}$

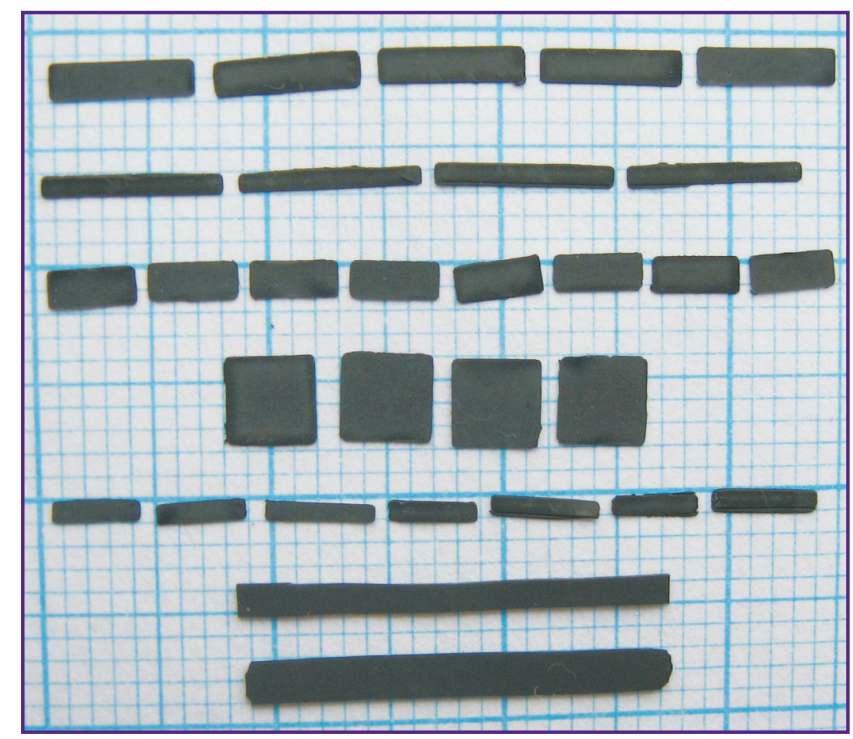

Figure 14. Experimental samples of endovitreal magnetic buckles (inner buckles)

The buckles of various sizes and shapes were made of silicone elastomer containing iron particles of $10 \mu \mathrm{m}$ in diameter; these ferromagnetic particles were coated with pure silicone and thus isolated from the eye tissues

particles from contacting the eye tissues (Figure 14). The implants can be easily implanted via an injector through 20G pars plana incision, are sturdy enough and aren't being damaged during implantation and manipulations. We injected up to 10 inner endovitreal magnetic buckles one by one and laid them along the edge of retinal breaks artificially created with vitreotome. 
Buckles formed continuous curved paths of arbitrary shape enclosing retinal breaks of any shape and size.

\section{Results}

The experiments performed have proved that it is technically possible to incorporate magnetic properties into conventional scleral buckle routinely used for episcleral buckling without changing its size and other mechanical properties. Modified buckle was visually indistinguishable from the conventional one and introduced no changes of surgical procedure. Suturing of the magnetic buckles was as easy as of standard scleral buckle. Endovitreal implants made of magnetoactive elastomer fitted well onto the retina, smoothly, tightly and effectively supported it pressing against the pigment epithelium with no signs of excessive pressure. Endovitreal buckles layed on retinal surface smoothly causing no rough impressions or tissue deformations.

Trying buckles of various sizes in experimental surgeries it was found out that the most optimal for implementation would be buckles no longer than $5 \mathrm{~mm}$. Surgical manipulations with longer buckles were complicated. Relatively short fragments $3-5 \mathrm{~mm}$ long allowed completion of all the manipulations required. Positioning inner buckles one after another it was possible to form a continuous path of hermetically fixated retina. After the surgery buckles could be readily removed with $25 \mathrm{G}$ vitreotome.

Thus considering the previously mentioned aspects of silicone oil tamponade hydrostatics it can be easily figured out which areas of retina are well supported being pressed against the pigment epithelium during silicone tamponade, which areas will experience just water medium displacement and which areas will experience no tamponade effect at all. In the insecure retinal areas additional mechanical support of the retina may be implemented once needed. The new technique proposed allows effective support of the retina by force of magnetic interactions. Magnetic scleral buckle may be employed not only in combination with endovitreal (inner) magnetic buckles applied on retina but also alone (e.g. when only scleral buckling without vitrectomy is planned). Modified buckle in this case exerts no additional forces on eye compared to the conventional buckle. Magnetic buckles perform all the functions of a standard buckle and in case of scleral buckling yields successful attachment of the retina magnetic properties of the buckle won't be used. But if pathologic process that caused retinal detachment continues to develop in postoperative period, surgeon will have an opportunity to employ additional options for mechanical support of retina using endovitreal buckles. Thus in some future it's possible that all the buckles will be manufactured impregnated with magnets: in this case surgeon will always be protected from possible complications as he will always have an opportunity of additional support of the retina in postoperative period (if needed) as well as during primary surgery.

\section{Conclusion}

Well known situation when light silicone oil floats upwards in the vitreous cavity leaving lower retina without tamponade effect while heavy silicone oil leaves upper retina with no tamponade effect suggests additional support of retina in the areas needing it with magnetic scleral buckling as a tool potentially able to increase success rate in retinal detachment surgical treatment.

While in most cases traditional surgical techniques of retinal detachment treatment may be quite sufficient, in some complicated cases mechanical support may be employed as a safe additional or optional measure. Combined with conventional techniques mechanical support may help patients in which it would be difficult if possible to achieve reattachment of the retina.

Acknowledgments. The authors are grateful to A.V. Gorshkov and N.R. Valetova of CJSC MedSil for their assistance in preparing the magnetic buckles.

Research funding. This work was supported by the Russian Science Foundation (project No.16-15-00208).

Conflict of interest. The authors have no conflict of interests.

\section{References}

1. Fawcett I.M., Williams R.L., Wong D. Contact angles of substances used for internal tamponade in retinal detachment surgery. Graefes Arch Clin Exp Ophthalmol 1994; 232(7): 438444, https://doi.org/10.1007/bf00186587.

2. Rush R., Sheth S., Surka S., Ho I., Gregory-Roberts J. Postoperative perfluoro-n-octane tamponade for primary retinal detachment repair. Retina 2012; 32(6): 1114-1120, https://doi. org/10.1097/iae.0b013e31822f56f6.

3. Eames I., Angunawela R.I., Aylward G.W., Azarbadegan A. A theoretical model for predicting interfacial relationships of retinal tamponades. Invest Ophthalmol Vis Sci 2010; 51(4): 2243-2247, https://doi.org/10.1167/iovs. 09-4442.

4. Wong D., Cazabon S., Ali H., Kumar I., Valldeperas X., Groenewald C., Pearce I. Can the sequential use of conventional silicone oil and heavy oil be a strategy for the management of proliferative vitreoretinopathy? Ann Acad Med Singapore 2006; 35(3): 181-184.

5. Schaal S., Sherman M.P., Barr C.C., Kaplan H.J. Primary retinal detachment repair: comparison of 1-year outcomes of four surgical techniques. Retina 2011; 31(8): 1500-1504, https://doi.org/10.1097/iae.0b013e31820d3f55.

6. Mehta S., Blinder K.J., Shah G.K., Grand M.G. Pars plana vitrectomy versus combined pars plana vitrectomy and scleral buckle for primary repair of rhegmatogenous retinal detachment. Can J Ophthalmol 2011; 46(3): 237-241, https:// doi.org/10.1016/j.jcjo.2011.05.003.

7. Storey P., Alshareef R., Khuthaila M., London N., Leiby B., DeCroos C., Kaiser R.; Wills PVR Study Group. Pars 
plana vitrectomy and scleral buckle versus pars plana vitrectomy alone for patients with rhegmatogenous retinal detachment at high risk for proliferative vitreoretinopathy. Retina 2014; 34(10): 1945-1951, https://doi.org/10.1097/iae.0000000000000216.

8. Rush R., Simunovic M.P., Sheth S., Chang A., Hunyor A.P. 23-gauge pars plana vitrectomy versus scleral buckling versus combined pars plana vitrectomy-scleral buckling for medium-complexity retinal detachment repair. Asia Pac J Ophthalmol 2014; 3(4): 215-219, https://doi.org/10.1097/ apo.0000000000000013.

9. Makarova L.A., Nadzharyan T.A., Alekhina Y.A., Stepanov G.V., Kazimirova E.G., Perov N.S., Kramarenko E.Yu. Magnetoactive elastomer as an element of a magnetic retina fixator. Smart Materials and Structures 2017; 26(9): 095054, https://doi.org/10.1088/1361-665x/aa82e9.

10. Puustjärvi T.J., Teräsvirta M.E. Retinal fixation of traumatic retinal detachment with metallic tacks: a case report with 10 years' follow-up. Retina 2001; 21(1): 54-56, https://doi. org/10.1097/00006982-200102000-00009.

11. Burke J.M., McDonald H.R., Neuwirth J., Lewandowski M.
Titanium retinal tacks with pneumatic insertion. Histologic evaluation in rabbits. Arch Ophthalmol 1987; 105(3): 404-408, https://doi.org/10.1001/archopht.1987.01060030124041.

12. Ohira A., de Juan E., Tsai M. Long-term histologic and electrophysiologic evaluation of the alloy retinal tack. Graefes Arch Clin Exp Ophthalmol 1991; 229(1): 95-98, https://doi. org/10.1007/bf00172270.

13. Tripathi R.C., Pon D.M., Levine R.A., Tripathi B.J., Falckh R.C., Moffat K.P. Retinal tacks: tolerance and tissue reaction in a human eye. Ophthalmic Surg 1989; 20(9): 658-662.

14. de Juan E. Jr., McCuen B.W., Machemer R. Mechanical retinal fixation using tacks. Ophthalmology 1987; 94(4): 337340, https://doi.org/10.1016/s0161-6420(87)33441-4.

15. Takhchidi Kh.P., Kazaykin V.N. Suture fixation of the retina in giant tears and abruptions of retina from the dentate line with the use of perfluororganic compounds. Oftal'mokhirurgiya 1996; 4: 23-28.

16. Pashtaev N.P., Grigorieva I.N. The first experience of microinvasive suture fixation of giant tears and abruptions of retina. Oftal'mokhirurgiya 2012; 1: 58-61. 